\title{
OTONEUROLOGICAL MANIFESTATION IN SUBCLAVIAN STEAL SYNDROME
}

\author{
Vinod Porwal, Anand Verma
}

1. Associate Professor. Department of Medicine, Sri Aurobindo Medical College and PG Institute, Indore.

2. Associate Professor. Department of Medicine, Sri Aurobindo Medical College and PG Institute, Indore.

\section{CORRESPONDING AUTHOR:}

Vinod Porwal,

5/15, Vijay Nagar, scheme 54,

Indore, Madhya Pradesh,

E-mail: vinporwal@yahoo.co.in

Ph: 00919926083487.

ABSTRACT: -The subclavian steal syndrome is characterized by a subclavian artery stenosis located proximal to the origin of the vertebral artery. In this case, the subclavian artery steals reverse-flow blood from the vertebrobasilar artery circulation to supply the arm during exertion, resulting in vertebrobasilar insufficiency. As the vertebrobasilar arterial system feeds both the peripheral and central auditory and vestibular systems. In subclavian steal syndrome, neurotological symptoms are expected because of the vertebrobasilar insufficiency. In this report, we describe a patient suffering from subclavian steal syndrome, who presented with isolated dizziness, recurrent vertigo, on moving around, while sitting no complaints were there and no postural vertigo was seen. Upon magnetic resonance imaging no ischaemic lesions were observed, suggesting that the central auditory and vestibular system are more likely to be involved in the pathogenesis of neurotological symptoms in subclavian steal syndrome. Patients complaining of numbness of the upper arm and isolated neurotological symptoms should be thoroughly examined for subclavian steal syndrome. Furthermore, regular follow-up must be undertaken in order to prevent other neurological deficits in the vertebrobasilar arterial territory.

KEYWORDS: Vertigo, Sensorineural hearing loss, Subclavian steal syndrome, Vertebrobasilar insufficiency

INTRODUCTION: The subclavian steal syndrome (SSS) refers to a vascular disorder in which there is occlusion or stenosis of the subclavian artery proximal to the vertebral artery origin (which is the subclavian artery) leads to altered vascular haemodynamics resulting in retrograde blood flow in the ipsilateral vertebral artery toward the upper arm, distal to the subclavian artery narrowing, where decreased blood pressure had been established. Subclavian artery lesions are usually asymptomatic because of the abundant collateral blood supply in the head, neck, and shoulder. However, these lesions produce neurologic symptoms when compensatory flow to the subclavian artery from the vertebral artery diverts too much flow toward the arm and away from intra-cranial structures leading to vertebrobasilar insufficiency (VBI) ${ }^{1}$ for example during arm swinging. The most important collateral circulation to the posterior fossa is through the circle of Willis, principally through the posterior communicating artery. When this communication is absent or inadequate, possibly due to concurrent extracranial carotid stenosis, then vertebrobasilar symptoms manifest.

The rate of SSS is estimated at 1.3\% (324 cases in 25,000 persons) in European patients referred for carotid and vertebral artery Doppler ultrasound, most frequently in Caucasians 
because of the increased incidence of atherosclerosis in this population. SSS generally occurs in patients $>55$ years of age and has a 2:1 male-to-female ratio ${ }^{2}$. Clinically, SSS may be suspected when the difference in blood pressure between the two arms exceeds $20 \mathrm{mmHg}$, but the diagnosis is essentially confirmed by Doppler ultrasound, although magnetic resonance imaging (MRI) with or without magnetic resonance angiography (MRA), computed tomography (CT) scan of the brain and digital subtraction angiography can also be used. Symptomatic patients require surgical intervention such as carotid-subclavian bypass, axillo-axillary bypass, or percutaneous transluminal angioplasty of the subclavian artery with stent placement. Thus, the goals of surgical treatment consist in restoration of the antegrade vertebral artery flow, alleviation of cerebral hypoperfusion, and improvement of arterial perfusion to the upper arm ${ }^{3}$. Moreover, aggressive management of risk factors, such as hypertension, diabetes and tobacco use, is also essential for successful treatment of this syndrome ${ }^{3}$.

The present report deals with a patient suffering from SSS, who was admitted to our unit on account of neurotological symptoms.

CASE REPORT: A 54 -year-old male, admitted with complaint of , unsteadiness and instability especially while walking, which still persists to this day on examination left radial was feeble no nystagmus, no cerebellar signs, rhombergs sign was negative, symptoms increased on active movement of left hand. He had no auditory disturbance or tinnitus. As the pulsations were feeble on left radial artery, CT Angio and Digital subtraction angiography done which revealed SSS on the left side, stenosis at the origin of the left vertebral artery and stenosis of both the carotid bifurcations (fig. 1). MRI didn't show any ischaemic lesions in the area of the brainstem (Fig.2). Work up was done to rule out takayasus arteritis was normal, even angiography findings were not consistent with it. Patient underwent carotid-subclavian bypass , left radial pulsation improved, but still his giddiness didn't improved literature was searched which showed coexistence of neurotological symptoms associated with SSS, there are few case reports which have reported it.

At neurotological examination, when performing Unterberger testing, he deviated towards the left side., there was no spontaneous nystagmus, but in the position of Rose (supine position, head hanging down and rotated to the left, middle, right, with eyes closed), a positional vertical up-beating nystagmus was present . Caloric testing was normal on both sides.

DISCUSSION: In SSS, occlusion or marked stenosis are present, usually due to atherosclerosis, of either the subclavian or innominate artery proximal to the origin of the VA. The established difference of pressure between the subclavian and basilar artery may lead to a siphoning effect with blood flowing from the opposite VA across the basilar artery and down the VA on the occluded side ${ }^{1}$. This results in a steal blood phenomenon at the expense of the basilar artery, as blood flow is reversed to the ipsilateral occlusion VA, which leads to a hypoperfusion state in the vertebrobasilar arterial (VBA) system and thus vertebrobasilar insufficiency (VBI).

SSS is classified as asymptomatic, oligosymptomatic, if only neurological symptoms or upper limb ischaemia are present and complete when both symptoms are found ${ }^{6}$. When symptomatic, the SSS can be manifest with a variety of VBI symptoms such as headache, blurred vision, diplopia, impairment of consciousness, dysarthria, and facial paraesthesiae. Neurotological symptoms, such as vertigo, are also mentioned in VBI and are considered to be of central origin ${ }^{7-8}$. Our patient suffering from SSS and thus from VBI presented with unsteadiness , recurrent vertigo. Although VBI is related to disturbed haemodynamics in the posterior 
cerebral circulation, neurotological symptoms may be isolated, without any other neurological signs of brainstem ischaemia ${ }^{9}$.

In our case, a positional nystagmus was revealed. The positional nystagmus could be an isolated finding, in VBI, even if there were no other neurological signs ${ }^{11}$. Moreover, positional vertical down-beating nystagmus has already been described in VIB 12-13; positional vertical upbeating nystagmus is attributed to brainstem lesions located at the ponto-mesencephalic and ponto-medullary junctions 14; accordingly, in our case no ischaemic lesions were located in the low brainstem. Some studies have reported that the most common finding in VBI is the unilateral vestibular paresis to caloric testing ${ }^{19}$. In our study, the caloric response reduced; it is currently accepted that caloric testing remains normal in central lesions 10. Abnormal oculomotor testing, such as smooth pursuit, is frequently seen in VBI patients ${ }^{14}$, in our case, abnormal saccades were detected, indicating a central nervous system involvement.

In our patient, sensorineural hearing loss was demonstrated, unilateral in one and bilateral in the other. Unilateral hearing loss, attributable to VBI is not so frequent, estimated at approximately $20 \%$, according to Yamasoba et al. ${ }^{15}$ in a series of 70 patients suffering from VBI; bilateral, sudden sensorineural hearing loss, although rare, is also described in VBI, in isolation ${ }^{16}$ or accompanied by neurological symptoms ${ }^{17}$.

Since the VBA system feeds both the peripheral and central region of the auditory and vestibular systems, it is difficult, in SSS, to precisely localize the site of insult. The presence of positional vertical nystagmus and prolonged wave $\mathrm{V}$ and increased wave $\mathrm{I}-\mathrm{V}$ latency interval in ABRs, the finding of abnormal saccades and the radiological evidence of cerebral ischaemic lesions support the hypothesis that, in SSS, the central auditory and vestibular system is implicated in the pathogenesis of neurotological symptoms.

\section{REFERENCES:}

1. Baloh RW. Vascular disorders. In: Baloh RW, Honrubia V, editors. Clinical neurophysiology of the vestibular system. $3^{\text {rd }}$ edn. New York: Oxford University Press, 2001. p. 292-233-308.

2. Perler BA, Becker GJ. Vascular intervention: A clinical approach. London: Thieme; 1998. p. 474-5.

3. Henry M, Henry I, Klonaris C, Hugel M. Percutaneous transluminal angioplasty of the subclavian arteries. In: Henry M, Ohki T, Polydorou A, Strigaris K, Kiskinis D, editors. Angioplasty and stenting of the carotid and supra-aortic trunks. London: Springer; 2004. p. 655-6.

4. Pagnini P, Palmeri G, Cipparrone L. Bidirectional horizontal and tridimensional apogeotropicnystagmus: 2 semeiological realities. ActaOtorhinolaryngolItal 1992;12:4554.

5. Walke OM, Treasure RL. Coexistent ipsilateralsubclavian steal and thoracic outlet compression syndromes. J ThoracCardiovascSurg 1975;69:874-5.

6. Albera B, Morra B, Poli L. La sindrome da furtodellasucclavia. ActaOtorhinolaryngolItal 1985;5(Suppl 7):1-22.

7. Bruyn GW. Vertigo and vertebrobasilar insufficiency.ActaOtolaryngol (Stockh) 1988;460:128-34.

8. Inui H, Yoneyama K, Kitaoku Y, Nakane M, Ohue S, Yamanaka T, et al. Four cases of vertebrobasilar insufficiency.ActaOtolaryngol (Stockh) 1998;533(Suppl):46-50. 
9. Grad A, Baloh RW. Vertigo of vascular origin: Clinical and electronystagmographic features in 84 cases. Arch Neurol 1989;46:281-4.

10. Seo T, Tominaga S, Sakagami M. Relationship between neurological asymptomatic vertigo and the vertebrobasilar system as revealed by magnetic resonance angiography. ORL J OtorhinolaryngolRelat Spec 2000;62:63-7.

11. Rosengart A, Hedges TR $3^{\text {rd }}$, Teal PA, DeWitt LD, Wu JK, Caplan LR, et al. Intermittent downbeat nystagmus due to vertebral artery compression. Neurology 1993;43:216-8.

12. Jacobson DM, Corbett JJ. Downbeat nystagmus and dolichoectasia of the vertebrobasilar artery. J Neuroophthalmol 2002;22:150-1.

13. Hankey GJ, Silbert PL, Edis RH.Localising value of primary position upbeatingnystagmus.Aust N Z J Med 1987;17:333-5.

14. Corneva J, Benitez LD, Lopez-Rios G, Rabiela MT. Vestibular and oculomotor abnormalities in vertebrobasilar insufficiency. Ann OtolRhinolLaryngol 1980;89:370-6.

15. Yamasoba T, Kikuchi S, Higo R. Deafness associated with vertebrobasilar insufficiency. J NeurolSci 2001;187:69-75.

16. Lee H, Yi HA, Baloh RW. Sudden bilateral simultaneous deafness with vertigo as a sole manifestation of vertebrobasilar insufficiency. J NeurolNeurosurg Psychiatry 2003;74:539-41.

17. Sauvaget E, Kici S, Petelle B, Kania R, Chabriat H, Herman Ph, et al. Vertebrobasilar occlusive disorders presenting as sudden sensorineural hearing loss. Laryngoscope 2004;114:327-32.

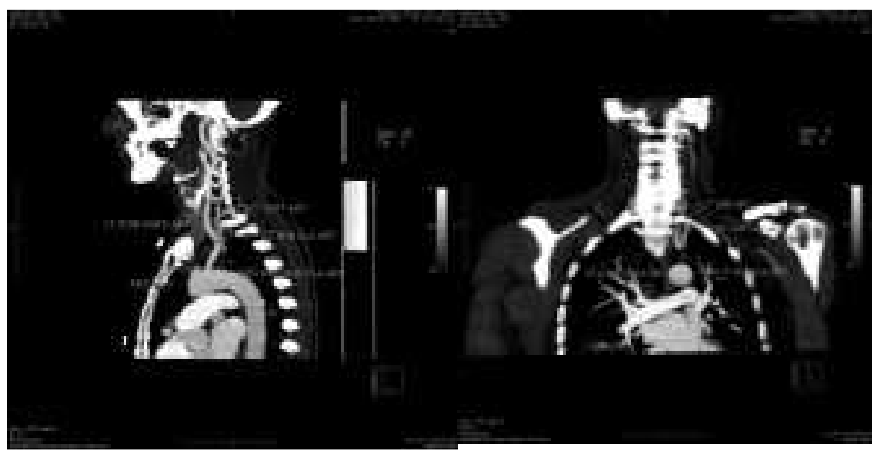

Fig 1a

fig $1 b$

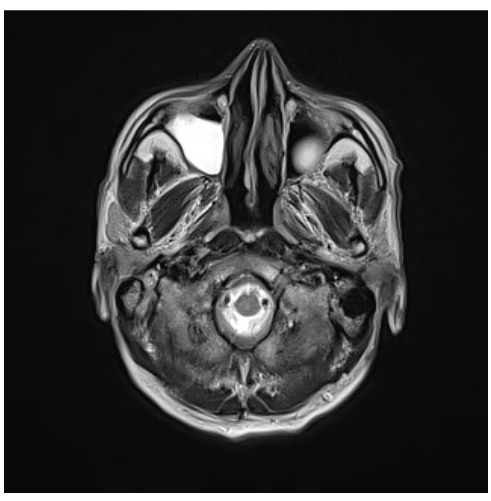

Fig. 2 Axial Gd-MRI scan of showing normal ( no ischaemic lesions) brainstem. 